\title{
On the shock-response-spectrum recursive algorithm of Kelly and Richman
}

\author{
Justin N. Martin ${ }^{1}$, Andrew J. Sinclair* and Winfred A. Foster \\ Aerospace Engineering Department, Auburn University, Auburn, AL, USA
}

Received 7 January 2010

Revised 16 June 2010

\section{Introduction}

The monograph "Principles and Techniques of Shock Data Analysis" written by Kelly and Richman in 1969 has become a seminal reference on the shock response spectrum (SRS) [1]. Because of its clear physical descriptions and mathematical presentation of the SRS, it has been cited in multiple handbooks on the subject $[2,3]$ and research articles [4-10]. Because of continued interest, two additional versions of the monograph have been published: a second edition by Scavuzzo and Pusey in 1996 [11] and a reprint of the original edition in 2008 [12]. The main purpose of this note is to correct several typographical errors in the manuscript's presentation of a recursive algorithm for SRS calculations. These errors are consistent across all three editions of the monograph. The secondary purpose of this note is to present a Matlab implementation of the corrected algorithm.

\section{Continuous-time solution}

The shock response spectrum considers the response of a single degree-of-freedom damped mechanical oscillator to a base excitation. The motion of the oscillator is $y(t)$, and the motion of the base is $x(t)$. This system obeys the following equation of motion.

$$
\ddot{y}+2 \zeta \omega_{n}(\dot{y}-\dot{x})+\omega_{n}^{2}(y-x)=0
$$

Here, $\omega_{n}$ is the undamped natural frequency of the oscillator, and $\zeta$ is the damping ratio. Accelerometer data for the base excitation, however, provides knowledge of $\ddot{x}$ not $x$ or $\dot{x}$. Therefore, it is convenient to consider the relative motion is $\xi(t)=y-x$ and the relative equation of motion.

$$
\ddot{\xi}+2 \zeta \omega_{n} \dot{\xi}+\omega_{n}^{2} \xi=-\ddot{x}
$$

For arbitrary initial conditions, $\xi_{0}$ and $\dot{\xi}_{0}$, Eq. (2) has the following solution.

$$
\begin{aligned}
\xi(t)=e^{-\zeta \omega_{n} t} & {\left[\xi_{0}\left(\cos \omega_{d} t+\frac{\zeta}{\sqrt{1-\zeta^{2}}} \sin \omega_{d} t\right)+\frac{\dot{\xi}_{0}}{\omega_{d}} \sin \omega_{d} t\right] } \\
& -\frac{1}{\omega_{d}} \int_{0}^{t} \ddot{x}(\tau) e^{-\zeta \omega_{n}(t-\tau)} \sin \omega_{d}(t-\tau) \mathrm{d} \tau
\end{aligned}
$$

\footnotetext{
*Corresponding author. Tel.: +1 334844 6825; Fax: +1 334844 6803; E-mail: sinclair@auburn.edu.

${ }^{1}$ Current affiliation: Aviation Engineering Directorate, U.S. Army Aviation and Missile Research Development and Engineering Center, Redstone Arsenal, AL, USA.
} 
Here, $\omega_{d}=\omega_{n} \sqrt{1-\zeta^{2}}$. Differentiation gives the solution for the relative velocity.

$$
\begin{aligned}
\dot{\xi}(t)= & e^{-\zeta \omega_{n} t}\left[-\xi_{0} \frac{\omega_{n}}{\sqrt{1-\zeta^{2}}} \sin \omega_{d} t+\dot{\xi}_{0}\left(\cos \omega_{d} t-\frac{\zeta}{\sqrt{1-\zeta^{2}}} \sin \omega_{d} t\right)\right] \\
& -\int_{0}^{t} \ddot{x}(\tau) e^{-\zeta \omega_{n}(t-\tau)}\left[\cos \omega_{d}(t-\tau)-\frac{\zeta}{\sqrt{1-\zeta^{2}}} \sin \omega_{d}(t-\tau)\right] \mathrm{d} \tau
\end{aligned}
$$

Several definitions exist for the SRS, differing in the ways the response is characterized [1]. For a definition based on the relative displacement of the oscillator, the solution in Eq. (3) is sufficient. The focus here, however, will be on another definition, based on the absolute acceleration of the oscillator. The absolute acceleration can be reconstructed using the relative position and velocity.

$$
\ddot{y}(t)=\ddot{\xi}+\ddot{x}=-2 \zeta \omega_{n} \dot{\xi}(t)-\omega_{n}^{2} \xi(t)
$$

Equations (3) and (4) correspond with Eqs (4.21) and (4.27), respectively, in Kelly and Richman; however, they reflect several errors in the original source.

\section{Discrete-data approximation}

In practice, evaluating Eqs (3) and (4) requires several considerations. First, the input accelerations are often only available at discrete time instants, and the output accelerations may only be desired at the same instants. The data points are here considered to be separated in time by a regular interval $\Delta t$. Next, to avoid evaluating the integrals in Eqs (3) and (4) over $\tau=0 \rightarrow t$ for each instant in time, a recursive algorithm is desired. The solutions for $\xi_{k} \equiv \xi\left(t_{k}\right)$ and $\dot{\xi}_{k} \equiv \dot{\xi}\left(t_{k}\right)$ are treated as initial conditions in calculating $\xi_{k} \equiv \xi\left(t_{k+1}\right)$ and $\dot{\xi}_{k} \equiv \dot{\xi}\left(t_{k+1}\right)$, and the integrals only need to be evaluated over $\tau=0 \rightarrow \Delta t$.

$$
\begin{aligned}
\xi_{k+1}= & e^{-\zeta \omega_{n} \Delta t}\left[\xi_{k}\left(\cos \omega_{d} \Delta t+\frac{\zeta}{\sqrt{1-\zeta^{2}}} \sin \omega_{d} \Delta t\right)+\frac{\dot{\xi}_{k}}{\omega_{d}} \sin \omega_{d} \Delta t\right] \\
& -\frac{1}{\omega_{d}} \int_{0}^{\Delta t} \ddot{x}\left(t_{k}+\tau\right) e^{-\zeta \omega_{n}(\Delta t-\tau)} \sin \omega_{d}(\Delta t-\tau) \mathrm{d} \tau \\
\dot{\xi}_{k+1}= & e^{-\zeta \omega_{n} \Delta t}\left[-\xi_{k} \frac{\omega_{n}}{\sqrt{1-\zeta^{2}}} \sin \omega_{d} \Delta t+\dot{\xi}_{k}\left(\cos \omega_{d} \Delta t-\frac{\zeta}{\sqrt{1-\zeta^{2}}} \sin \omega_{d} \Delta t\right)\right] \\
& -\int_{0}^{\Delta t} \ddot{x}\left(t_{k}+\tau\right) e^{-\zeta \omega_{n}(\Delta t-\tau)}\left[\cos \omega_{d}(\Delta t-\tau)-\frac{\zeta}{\sqrt{1-\zeta^{2}}} \sin \omega_{d}(\Delta t-\tau)\right] \mathrm{d} \tau
\end{aligned}
$$

In order to evaluate the integral terms in Eqs (6) and (7) a continuous approximation of the discrete data needs to be formed. Kelly and Richman considered a parabolic approximation using the $(k-1), k$, and $(k+1)$ data points.

$$
\begin{aligned}
\ddot{x}\left(t_{k}+\tau\right) & =\ddot{x}_{k}+S_{k} \frac{\tau}{\Delta t}+\frac{S_{k-1}^{2}}{2}\left(\frac{\tau^{2}}{\Delta t^{2}}-\frac{\tau}{\Delta t}\right) \\
S_{k} & =\ddot{x}_{k+1}-\ddot{x}_{k} \\
S_{k-1}^{2} & =\ddot{x}_{k+1}-2 \ddot{x}_{k}+\ddot{x}_{k-1}
\end{aligned}
$$

Given a discrete time-history of accelerometer data, the resulting oscillator acceleration at any instant in time can now be calculated by substituting Eq. (8) into Eqs (6) and (7), and using the resulting values in Eq. (5). Evaluating Eqs (6) and (7) requires the calculation of the following Duhamel integrals.

$$
\begin{aligned}
I_{1} & \equiv \int_{0}^{\Delta t} e^{-\zeta \omega_{n}(\Delta t-\tau)} \sin \omega_{d}(\Delta t-\tau) \mathrm{d} \tau \\
& =\frac{\sqrt{1-\zeta^{2}}}{\omega_{n}}\left[1-e^{-\zeta \omega_{n} \Delta t}\left(\cos \omega_{d} \Delta t+\frac{\zeta}{\sqrt{1-\zeta^{2}}} \sin \omega_{d} \Delta t\right)\right]
\end{aligned}
$$




$$
\begin{aligned}
& I_{2} \equiv \int_{0}^{\Delta t} \tau e^{-\zeta \omega_{n}(\Delta t-\tau)} \sin \omega_{d}(\Delta t-\tau) \mathrm{d} \tau \\
& =\frac{1}{\omega_{n}^{2}}\left\{\omega_{d} \Delta t-2 \zeta \sqrt{1-\zeta^{2}}+e^{-\zeta \omega_{n} \Delta t}\left[2 \zeta \sqrt{1-\zeta^{2}} \cos \omega_{d} \Delta t-\left(1-2 \zeta^{2}\right) \sin \omega_{d} \Delta t\right]\right\} \\
& I_{3} \equiv \int_{0}^{\Delta t} \tau^{2} e^{-\zeta \omega_{n}(\Delta t-\tau)} \sin \omega_{d}(\Delta t-\tau) \mathrm{d} \tau \\
& =-\frac{1}{\omega_{n}^{3}}\left\{4 \zeta \omega_{d} \Delta t+\sqrt{1-\zeta^{2}}\left(2-8 \zeta^{2}-\omega_{n}^{2} \Delta t^{2}\right)\right. \\
& \left.+e^{-\zeta \omega_{n} \Delta t}\left[\left(8 \zeta^{2}-2\right) \sqrt{1-\zeta^{2}} \cos \omega_{d} \Delta t+\left(8 \zeta^{2}-6\right) \zeta \sin \omega_{d} \Delta t\right]\right\} \\
& I_{4} \equiv \int_{0}^{\Delta t} e^{-\zeta \omega_{n}(\Delta t-\tau)} \cos \omega_{d}(\Delta t-\tau) \mathrm{d} \tau \\
& =\frac{1}{\omega_{n}}\left[\zeta-e^{-\zeta \omega_{n} \Delta t}\left(\zeta \cos \omega_{d} \Delta t-\sqrt{1-\zeta^{2}} \sin \omega_{d} \Delta t\right)\right] \\
& I_{5} \equiv \int_{0}^{\Delta t} \tau e^{-\zeta \omega_{n}(\Delta t-\tau)} \cos \omega_{d}(\Delta t-\tau) \mathrm{d} \tau \\
& =\frac{1}{\omega_{n}^{2}}\left\{1-2 \zeta^{2}+\zeta \omega_{n} \Delta t-e^{-\zeta \omega_{n} \Delta t}\left[\left(1-2 \zeta^{2}\right) \cos \omega_{d} \Delta t+2 \zeta \sqrt{1-\zeta^{2}} \sin \omega_{d} \Delta t\right]\right\} \\
& I_{6} \equiv \int_{0}^{\Delta t} \tau^{2} e^{-\zeta \omega_{n}(\Delta t-\tau)} \cos \omega_{d}(\Delta t-\tau) \mathrm{d} \tau \\
& =\frac{1}{\omega_{n}^{3}}\left\{2 \zeta\left(4 \zeta^{2}-3\right)+2\left(1-2 \zeta^{2}\right) \omega_{n} \Delta t+\zeta \omega_{n}^{2} \Delta t^{2}\right. \\
& \left.-e^{-\zeta \omega_{n} \Delta t}\left[2 \zeta\left(4 \zeta^{2}-3\right) \cos \omega_{d} \Delta t+2 \sqrt{1-\zeta^{2}}\left(1-4 \zeta^{2}\right) \sin \omega_{d} \Delta t\right]\right\}
\end{aligned}
$$

Using Eqs (9-14), the solutions for $\xi_{k+1}$ and $\dot{\xi}_{k+1}$ can now be rewritten as shown.

$$
\begin{aligned}
& \xi_{k+1}=B_{1} \xi_{k}+B_{2} \dot{\xi}_{k}+B_{3} \ddot{x}_{k}+B_{4} S_{k}+B_{5} S_{k-1}^{2} \\
& \frac{\dot{\xi}_{k+1}}{\omega_{n}}=B_{6} \xi_{k}+B_{7} \dot{\xi}_{k}+B_{8} \ddot{x}_{k}+B_{9} S_{k}+B_{10} S_{k-1}^{2}
\end{aligned}
$$

Here, the $B$ coefficients are defined using Eqs (6) and (7) and the definitions of $I_{1}$ through $I_{6}$.

$$
\begin{aligned}
& B_{1}=e^{-\zeta \omega_{n} \Delta t}\left(\cos \omega_{d} \Delta t+\frac{\zeta}{\sqrt{1-\zeta^{2}}} \sin \omega_{d} \Delta t\right) \\
& B_{2}=\frac{e^{-\zeta \omega_{n} \Delta t}}{\omega_{d}} \sin \omega_{d} \Delta t \\
& B_{3}=-\frac{I_{1}}{\omega_{d}}
\end{aligned}
$$




$$
\begin{aligned}
& B_{4}=-\frac{I_{2}}{\omega_{d} \Delta t} \\
& B_{5}=-\frac{1}{2 \omega_{d}}\left(\frac{I_{3}}{\Delta t^{2}}-\frac{I_{2}}{\Delta t}\right) \\
& B_{6}=-\frac{e^{-\zeta \omega_{n} \Delta t}}{\sqrt{1-\zeta^{2}}} \sin \omega_{d} \Delta t \\
& B_{7}=\frac{e^{-\zeta \omega_{n} \Delta t}}{\omega_{n}}\left(\cos \omega_{d} \Delta t-\frac{\zeta}{\sqrt{1-\zeta^{2}}} \sin \omega_{d} \Delta t\right) \\
& B_{8}=\frac{\zeta I_{1}}{\omega_{d}}-\frac{I_{4}}{\omega_{n}} \\
& B_{9}=\frac{\zeta I_{2}}{\omega_{d} \Delta t}-\frac{I_{5}}{\omega_{n} \Delta t} \\
& B_{10}=\frac{\zeta}{2 \omega_{d}}\left(\frac{I_{3}}{\Delta t^{2}}-\frac{I_{2}}{\Delta t}\right)-\frac{1}{2 \omega_{n}}\left(\frac{I_{6}}{\Delta t^{2}}-\frac{I_{5}}{\Delta t}\right)
\end{aligned}
$$

Finally, the expressions for these coefficients can be simplified using Eqs (9-14).

$$
\begin{aligned}
B_{1}= & e^{-\zeta \omega_{n} \Delta t}\left(\cos \omega_{d} \Delta t+\frac{\zeta}{\sqrt{1-\zeta^{2}}} \sin \omega_{d} \Delta t\right) \\
B_{2}= & \frac{e^{-\zeta \omega_{n} \Delta t}}{\omega_{d}} \sin \omega_{d} \Delta t \\
B_{3}= & -\frac{1}{\omega_{n}^{2}}\left(1-B_{1}\right) \\
B_{4}= & -\frac{1}{\omega_{n}^{2}}\left[1-\frac{2 \zeta}{\omega_{n} \Delta t}\left(1-e^{-\zeta \omega_{n} \Delta t} \cos \omega_{d} \Delta t\right)-\frac{\left(1-2 \zeta^{2}\right) e^{-\zeta \omega_{n} \Delta t} \sin \omega_{d} \Delta t}{\omega_{d} \Delta t}\right] \\
B_{5}= & -\frac{1}{2 \omega_{n}^{2}}\left\{-\frac{4 \zeta}{\omega_{n} \Delta t}-\left[\frac{2\left(1-4 \zeta^{2}\right)}{\omega_{n}^{2} \Delta t^{2}}-\frac{2 \zeta}{\omega_{n} \Delta t}\right]\left(1-e^{-\zeta \omega_{n} \Delta t} \cos \omega_{d} \Delta t\right)\right. \\
& \left.+\left(\frac{1-2 \zeta^{2}}{\omega_{n} \Delta t}+\frac{2 \zeta\left(3-4 \zeta^{2}\right)}{\omega_{n}^{2} \Delta t^{2}}\right) \frac{e^{-\zeta \omega_{n} \Delta t} \sin \omega_{d} \Delta t}{\sqrt{1-\zeta^{2}}}\right\} \\
B_{6}= & -\omega_{n} B_{2} \\
B_{7}= & \frac{e^{-\zeta \omega_{n} \Delta t}}{\omega_{n}}\left(\cos \omega_{d} \Delta t-\frac{\zeta}{\sqrt{1-\zeta^{2}}} \sin \omega_{d} \Delta t\right) \\
B_{8}= & -\frac{B_{2}}{\omega_{n}} \\
B_{9}= & \frac{B_{1}-1}{\omega_{n}^{3} \Delta t}
\end{aligned}
$$




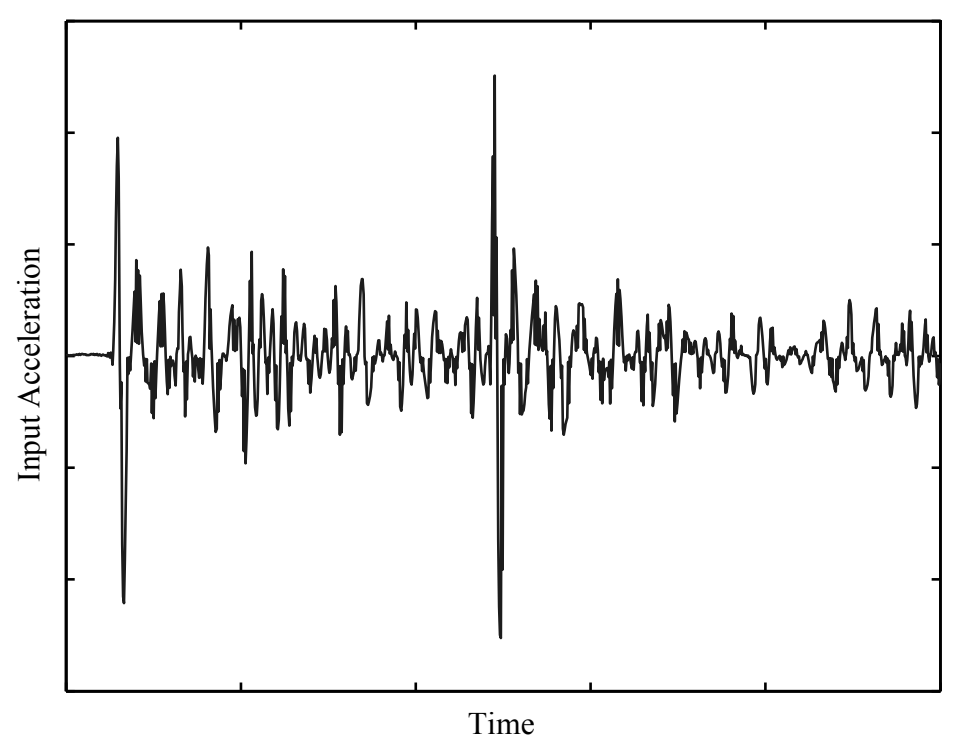

Fig. 1. Time history of sample input acceleration.

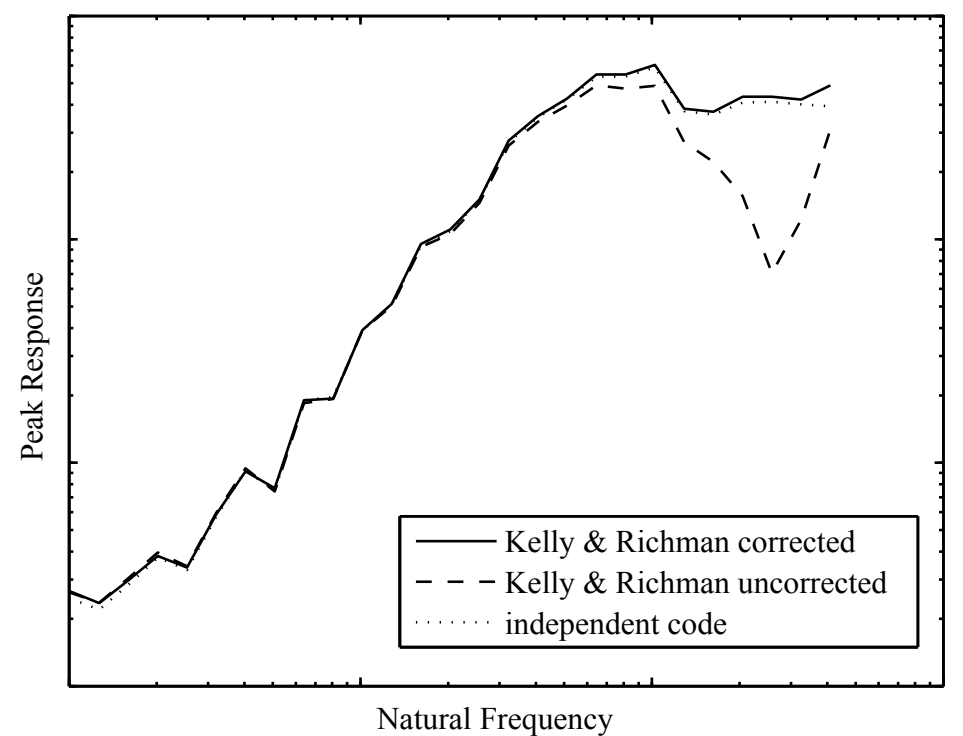

Fig. 2. SRS of input acceleration for $\zeta=0.05$ using three different algorithms.

$$
\begin{aligned}
B_{10}= & -\frac{1}{2 \omega_{n}^{2}}\left\{\frac{2}{\omega_{n} \Delta t}-\left(\frac{1}{\omega_{n} \Delta t}+\frac{4 \zeta}{\omega_{n}^{2} \Delta t^{2}}\right)\left(1-e^{-\zeta \omega_{n} \Delta t} \cos \omega_{d} \Delta t\right)\right. \\
& \left.-\left[\frac{2\left(1-2 \zeta^{2}\right)}{\omega_{n}^{2} \Delta t^{2}}-\frac{\zeta}{\omega_{n} \Delta t}\right] \frac{e^{-\zeta \omega_{n} \Delta t} \sin \omega_{d} \Delta t}{\sqrt{1-\zeta^{2}}}\right\}
\end{aligned}
$$

Equations (27-36) correspond with Eqs (6.58-67) in Kelly and Richman and Eqs (7.38-47) in Scavuzzo and Pusey; however, they reflect negative-sign errors in $B_{3}, B_{4}$, and $B_{10}$ in the original source. The corrected SRS algorithm for a sequence of input accelerations and a desired range of natural frequencies can be evaluated using Eqs (5), (15), (16), and (27-36). 


\section{Comparison and results}

The corrections described in the preceding paragraphs were verified by comparing the corrected algorithm and the original algorithm to an independent SRS code. The independent code used a piecewise-linear approximation for the base acceleration, as described by Paz [13]. The various algorithms were applied to accelerometer data from the ignition environment of live-fire testing of the Space Shuttle Reusable Solid Rocket Motor (RSRM). Specifically, data was evaluated from the radial channel at station 1479.5 on Technical Evaluation Motor 13. The data was sampled at $10,000 \mathrm{~Hz}$. The acceleration time history is shown in Fig. 1.

The SRS of this acceleration data is shown in Fig. 2 as calculated using three different algorithms. The corrected algorithm of Eqs (5), (15), (16), and (27-36) are compared with the uncorrected algorithm from Kelly and Richman as well as the independent code. For each algorithm, a damping ratio of $\zeta=0.05$ was used, and the peak response was calculated for a range of natural frequencies at one-third octaves up to the Nyquist frequency. The corrected algorithm and the independent code show strong agreement with each other; however, the uncorrected algorithm displays large differences in the high-frequency regime. The MATLAB implementation of the corrected algorithm is available for download at http://www.eng.auburn.edu/users/sinclaj/SRS/.

\section{Acknowledgements}

The authors are grateful to Robin Ferebee, Phillip Harrison, and Neil Murphy of NASA/Marshall Space Flight Center for access to the RSRM accelerometer data and the independent SRS code.

\section{References}

[1] R.D. Kelly and G. Richman, Principles and Techniques of Shock Data Analysis, The Shock and Vibration Information Center, Washington, D.C., $1969, \S 4.3 \& 6.6$.

[2] H. Himelblau, A.G. Piersol, J.H. Wise and M.R. Grundvig, Handbook for Dynamic Data Acquisition and Analysis, Chap. 5, Institute of Environmental Sciences and Technology, Rolling Meadows, Illinois, 1994.

[3] S. Rubin, "Concepts in Shock Data Analysis", Shock and Vibration Handbook, edited by C. M. Harris, Chap. 23, McGraw-Hill, New York, 4th ed., 1996.

[4] H.C. Pusey, "An Historical View of Dynamic Testing", The Journal of Environmental Sciences 20(5) (1977), 9-14.

[5] J.R. Houghton, "Shock Spectrum Ratios Applied to the Comparison of Pulse Signatures", Journal of Mechanical Design 102(1) (1980), 64-76.

[6] D.O. Smallwood, "An Improved Recursive Formula for Calculating Shock Response Spectra", Shock and Vibration Bulletin: Proceedings of the 51st Symposium on Shock and Vibration, San Diego, California, 1980.

[7] K. Peleg and S. Hinga, "Combining Fourier and Shock Spectra for Improved Test Specification", Journal of Sound and Vibration 110(2) (1986), 289-307.

[8] S. Smith and R. Melander, "Why Shock Measurements Performed at Different Facilities Don't Agree", Proceedings of the 66th Shock and Vibration Symposium, Biloxi, Mississippi, 1995.

[9] M. Sek, V. Rouillard, H. Tarash and S. Crawford, "Enhancement of Cushioning Performance with Paperboard Crumple Inserts", Packaging Technology and Science 18 (2005), 273-278.

[10] K. Shin and M.J. Brennan, "Two simple methods to suppress the residual vibrations of a translating or rotating flexible cantilever beam", Journal of Sound and Vibration 312 (2008), 140-150.

[11] R.J. Scavuzzo and H. Pusey, Principles and Techniques of Shock Data Analysis, The Shock and Vibration Information Analysis Center, Arvonia, Virginia, 2nd ed., 1996.

[12] R.D. Kelly and G. Richman, Shock Data Analysis - Engineering Principles and Techniques, Wexford Press, Eldersburg, Maryland, 2008.

[13] M. Paz, Structural Dynamics: Theory and Computation, Van Nostrand Reinhold, New York, 2nd ed., 1985, Sect. 4.4. 

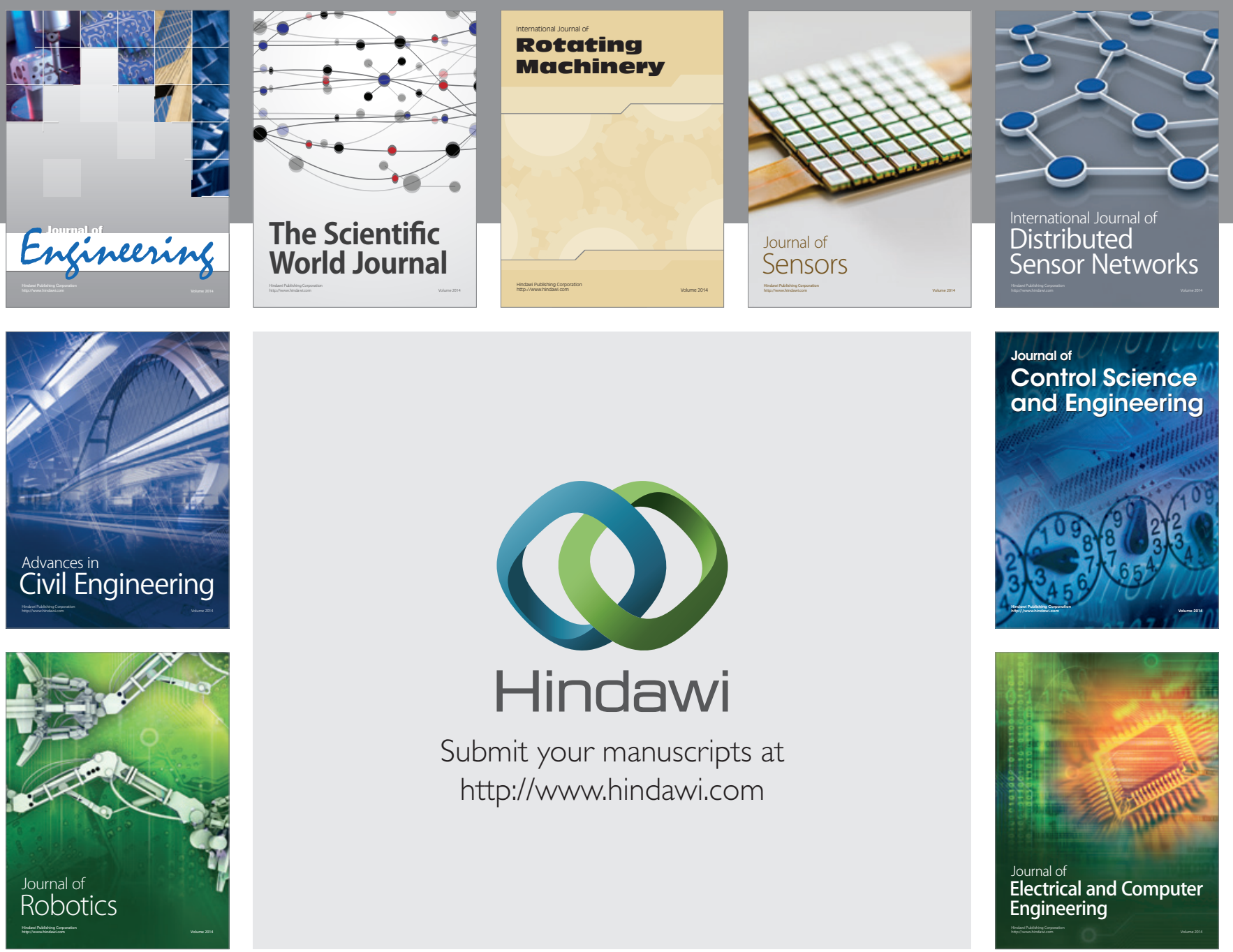

Submit your manuscripts at

http://www.hindawi.com
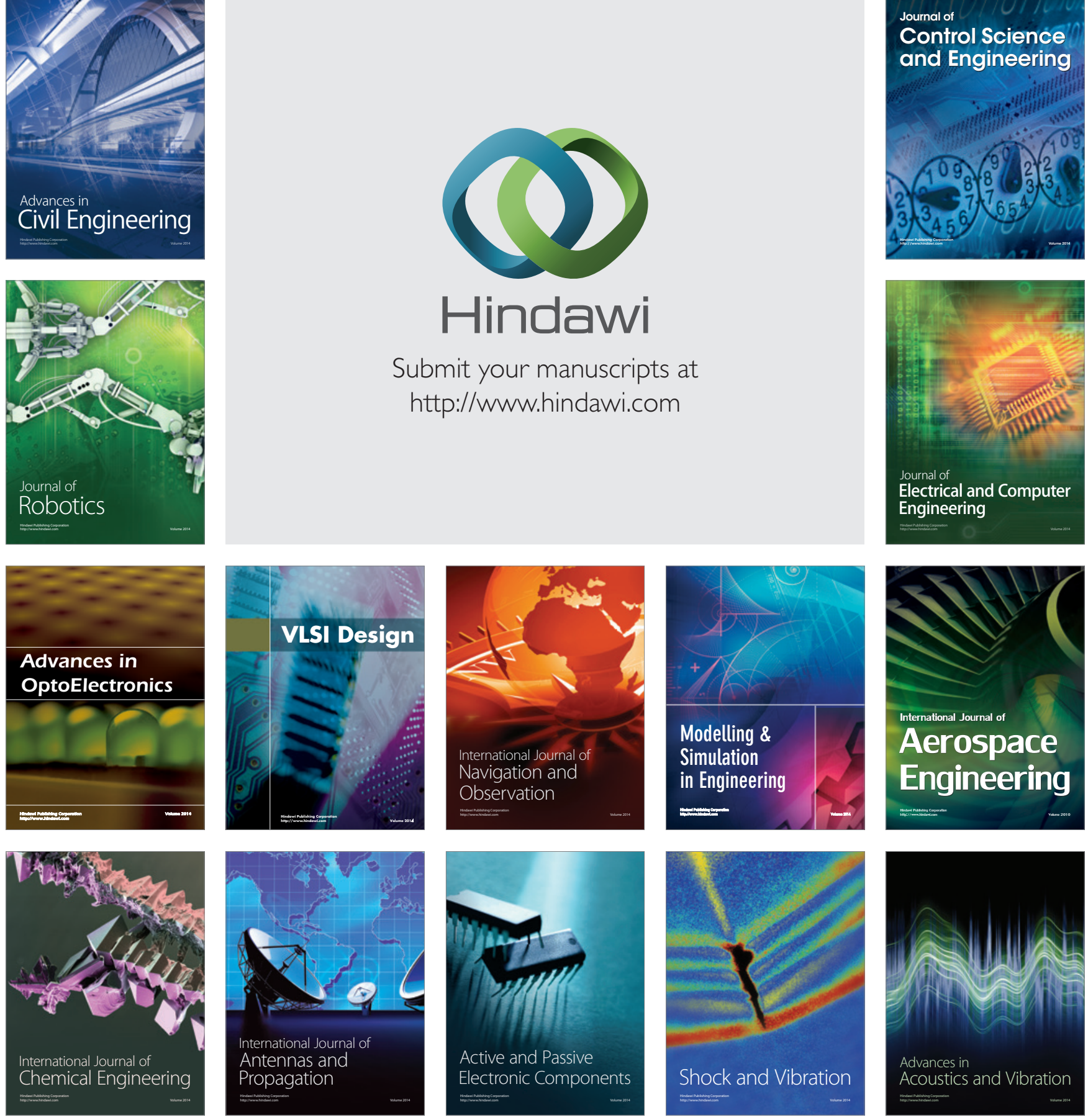